\title{
Effect of heat treatment on metallothionein isoforms using capillary zone electrophoresis
}

\author{
K. Kubo ${ }^{1}$, Y. Sakita ${ }^{1}$ and T. Minami ${ }^{2 *}$ \\ ${ }^{1}$ Faculty of Pharmaceutical Sciences, Kinki University, 3-4-1 Kowakae, Higashi-Osaka, Osaka 577-8502, Japan \\ ${ }^{2}$ Department of Living Sciences \& Program Processing, Toyo-Oka Junior College, Kinki University, 160 Tobera, \\ Toyo-Oka 668-8580, Japan
}

The effect of heat treatment on metallothionein (MT) isoforms was observed by using capillary zone electrophoresis (CZE) at a neutral $\mathrm{pH}$. Commercially available standard MT-1 preparation isolated from rabbit liver present as $\mathrm{Cd}$ and Zn mixed form was used as MT standard for the experiments in CZE. The MT-1 preparation was detected as two major peaks MT-1 and MT-2 identified from the migration times corresponded with those of purified isoforms and several minor peaks by CZE using a polyacrylamide-coated capillary at pH 7.4. The peak height of MT-1 of the standard MT-1 specimen decreased gradually from those of the non-heated specimen by heat treatment at $100{ }^{\circ} \mathrm{C}$ until $4 \mathrm{~min}$, but the peak of MT-2 did not decrease. In the cytosol fraction obtained from $\mathrm{Zn}$-injected mouse liver, many peaks including MT-1 and MT-2 were detected in the specimen without heat treatment. The specimen heated at $100^{\circ} \mathrm{C}$ for $15 \mathrm{~s}$ had a stable baseline and the peaks of both MT-1 and MT-2 were detected well. The peak heights of MT-1 and MT2 in cytosol fraction decreased gradually by heat treatment on time dependent manner. However, the ratio of peak heights of MT-1 and MT-2 did not change. From these results, the ratio of MT-1 and MT-2 in cytosol fraction is able to be observed well on CZE analysis after heat treatment.

\section{Introduction}

Metallothionein (MT) is an inducible protein, with a molecular mass of about 7,000. Although MT can bind with metals such as $\mathrm{Zn}, \mathrm{Cu}, \mathrm{Cd}$, and $\mathrm{Ag}, \mathrm{Zn}_{7}-\mathrm{MT}$ is commonly induced in the organs except the injection of $\mathrm{Cu}, \mathrm{Cd}$ or $\mathrm{Ag}$ [1]. MT exists as two major isoforms MT-1 and MT-2 in mammalian species, and both MTs vary by a few amino acid residues and have consequently very similar hydrophobicities and electric charges. Both MTs are induced coordinately in most organs by same stimuli $[1,2]$.

MT is known to be a heat stable protein, and $\mathrm{Zn}$ in $\mathrm{Zn}_{7^{-}}$ MT is easily replaced by $\mathrm{Cd}$ after the addition of $\mathrm{Cd}$ in vitro [2]. On the basis of the properties of MT, the quantification of MT in tissues is commonly calculated from $\mathrm{Cd}$ content in the solution after the addition of $\mathrm{Cd}$ and the heat treatment. The method is named as Cd-hem [3,4]. The outline of the Cd-hem method is as follows: Cd solution is added to a cytosol fraction obtained from tissue homogenate, and hemoglobin is added to the mixture solution to complex the excess of $\mathrm{Cd}$. The specimen is then heated at $100{ }^{\circ} \mathrm{C}$ for $1 \mathrm{~min}$ to precipitate selectively the Cd-bound hemoglobin. After centrifugation, the Cd concentration in the supernatant is measured by atomic absorption spectrometry. Then, the MT content in the tissues is calculated from the Cd concentration, on the basis of the stoechiometry of the totally metallated $\mathrm{Cd}$ complexes $\left(\mathrm{Cd}_{7}-\mathrm{MT}\right)$ encountered in 
mammalian tissues. The recovery rate of MT measured by the $\mathrm{Cd}$-hem method preformed best as well as radio immunoassay (RIA) method [5]. However, as heat treatment is a high stress for protein, the structure of MT is likely to change. No reports to date have described the effect of heat treatment on MT isoforms.

Capillary zone electrophoresis (CZE) is a powerful technique for the separation of the different forms of MTs, which have minor differences in terms of isoelectric points [6]. It associates rapid analysis, microsampling, and high resolution. As we previously succeeded in separating MT isoforms in cytosol fraction with CZE analysis using a polyacrylamide-coated capillary at a neutral pH [7], we tried to observe the effect of heat treatment on the properties of MT isoforms by using CZE analysis in the present study.

\section{Materials and methods}

\section{Materials}

N-2-Hydroxyethylpiperazin-N'-2-ethanesulfonic acid (HEPES) was purchased from Dojindo Laboratories (Kumamoto, Japan). Ammonium persulfate and tris (hydroxymethyl) aminomethane (Tris) were from Wako Pure Chemicals (Osaka, Japan). Rabbit liver MT-1 as standard was purchased from Sigma (St. Louis, MO, USA). All other chemicals were of analytical grade and obtained from Wako Pure Chemicals. The fused-silica capillary tube (i.d. $75 \mu \mathrm{m}$; o.d. $375 \mu \mathrm{m})$ was obtained from Otsuka Electronics Co. Ltd. (Osaka, Japan).

\section{CZE analysis}

CZE with a polyacrylamide-coated capillary was performed on a Quanta 4000 capillary electrophoresis system (Nihon Waters, Tokyo). The capillary was coated according to the previous report [6]. The electrophoresis was carried out with

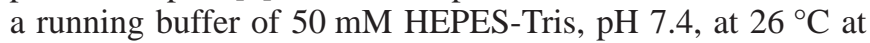
a constant voltage of $-20 \mathrm{kV}$ on a capillary with a total length of $33 \mathrm{~cm}$ (effective length of $25 \mathrm{~cm}$ ). The samples were introduced from the cathode end of the capillary for $6 \mathrm{sec}$ by hydrostatic injection $(10 \mathrm{mbar})$. Separations were monitored at $214 \mathrm{~nm}$.

\section{Preparation of mouse liver cytosol fraction}

Mice were subcutaneously injected $50 \mathrm{mg} / \mathrm{kg}$ of zinc in the form of zinc sulfate. Twenty-four hours after injection, the mice were decapitated and the livers were removed. The liver samples were frozen until required. The liver was homogenized to make a $10 \%$ homogenate with a $0.25 \mathrm{M}$ aqueous sucrose solution. The homogenate was immediately centrifugation at $700 \mathrm{xg}$ for $10 \mathrm{~min}$. The supernatant was centrifugation at $100,000 \mathrm{xg}$ for $30 \mathrm{~min}$, and the cytosol fraction was obtained.

\section{Heat treatment}

For observation of the effect of heat treatment on standard MT-1, $1 \mathrm{mg} / \mathrm{ml}$ of MT-1 specimen was incubated at $100{ }^{\circ} \mathrm{C}$ for 1,2 , and 4 minutes, and then centrifugation at $10,000 \mathrm{xg}$ for $10 \mathrm{~min}$. The supernatant was loaded onto the column for $6 \mathrm{~s}$ by hydrostatic injection (10 mbar). For observation of the effect of heat treatment on MT isoforms in cytosol fraction, the cytosol fraction obtained from $\mathrm{Zn}$-injected mouse liver was incubated at $100{ }^{\circ} \mathrm{C}$ for $15 \mathrm{~s}, 30 \mathrm{~s}, 1,2$, and $4 \mathrm{~min}$, and then centrifugation at $10,000 \mathrm{xg}$ for $10 \mathrm{~min}$. The supernatant was injected onto the separation capillary for $6 \mathrm{~s}$ by hydrostatic injection (10 mbar).

\section{Results}

The effect of heat treatment on standard MT-1 specimen is shown in figure 1. Standard MT-1 specimen was detected as ten and more peaks within $10 \mathrm{~min}$, and MT-2 was also observed in the specimen (Fig. 1a) from the migration times

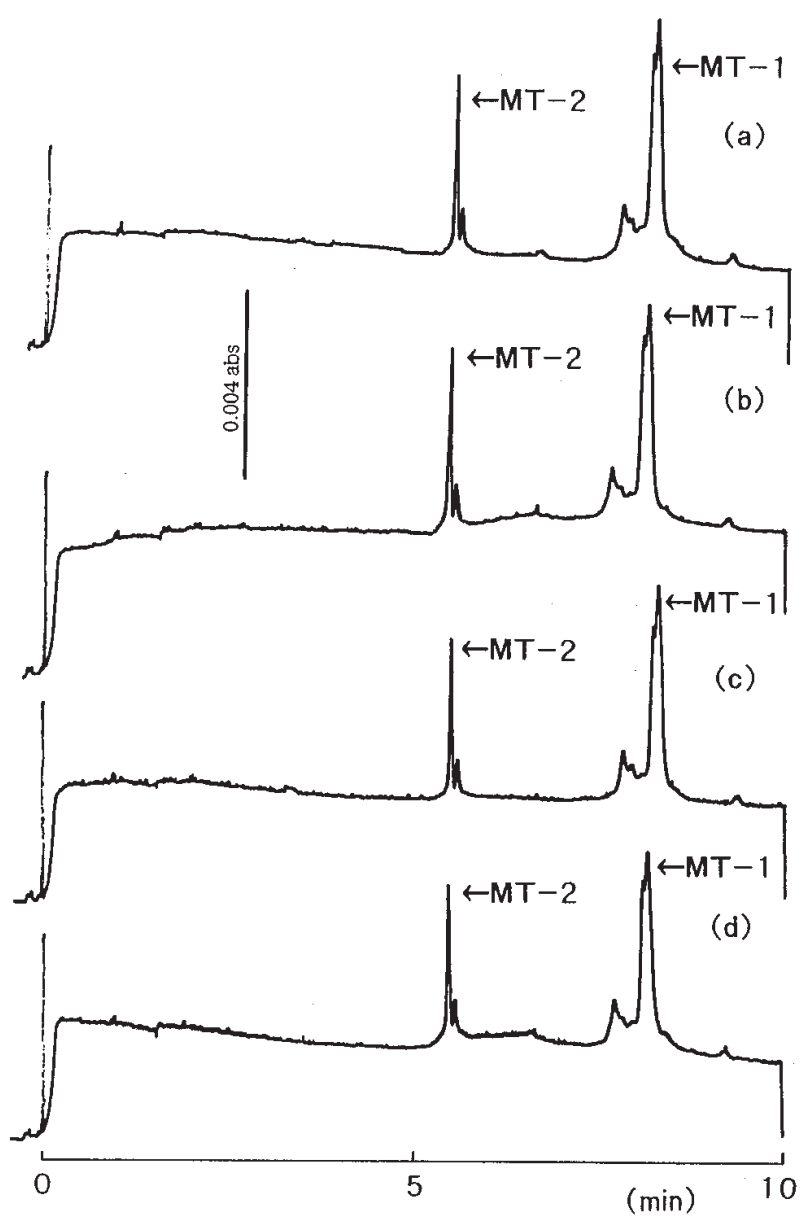

Figure 1. Effect of heat treatment on the electropherogram of standard MT-1 isoform. (a) Non-heated specimen. The specimen was incubated at $100{ }^{\circ} \mathrm{C}$ for $1 \mathrm{~min}$ (b), $2 \mathrm{~min}$ (c), and $4 \mathrm{~min}$ (d). 

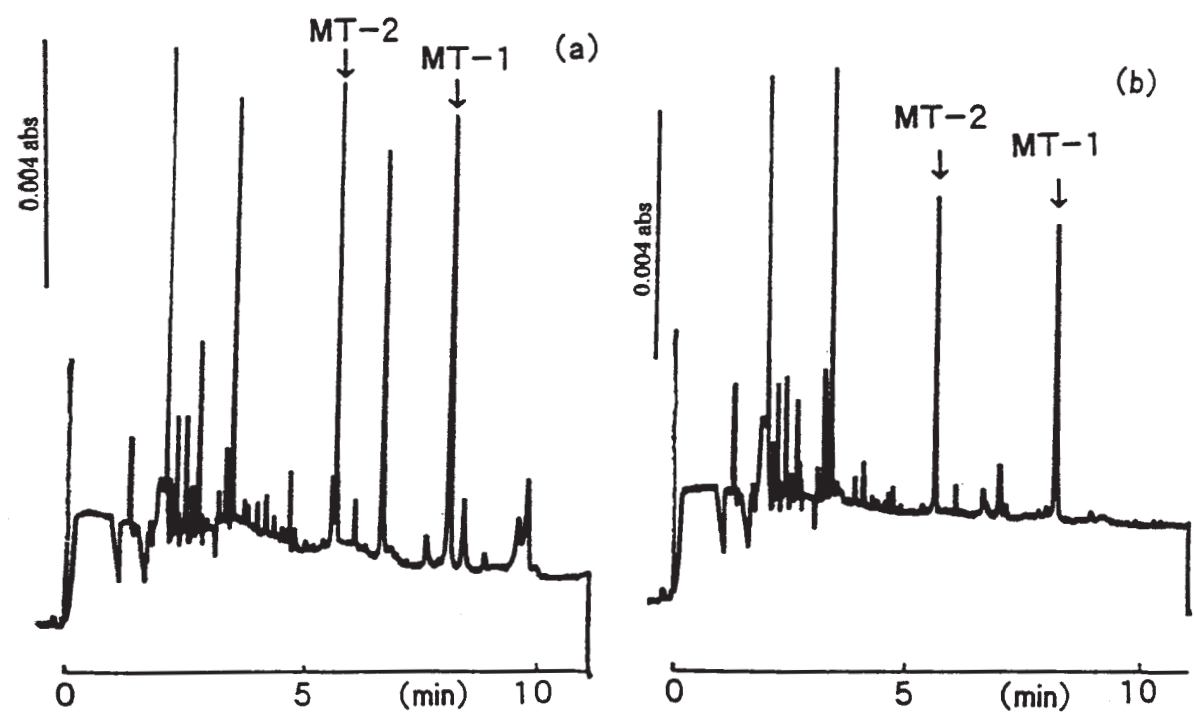

Figure 2. Effect of heat treatment on the electropherogram of the cytosol fraction of $\mathrm{Zn}$-injected mouse liver. The cytosol fraction was incubated at $100{ }^{\circ} \mathrm{C}$ for $15 \mathrm{~s} \mathrm{(a)}$, and $4 \mathrm{~min}(\mathrm{~b})$.

corresponded with those of purified MT-1 and MT-2 isoforms (MT-1: $7.6 \mathrm{~min}$; MT-2: $5.2 \mathrm{~min}$ ). Peak height of MT1 of specimen decreased gradually by heat treatment at $100{ }^{\circ} \mathrm{C}$ until $4 \mathrm{~min}$, but the peak of MT-2 did not decrease (Fig. 1b-d).

Heat treatment changed the peak heights of MT-1 and MT-2 in cytosol fraction as shown in figure 2. Non-heated cytosol fraction of $\mathrm{Zn}$-injected mouse liver showed a lot of peaks within $15 \mathrm{~min}$, but the peaks corresponded with those of MT-1 and MT-2 isoforms were observed from the migration times. The baseline was not stable, and both peaks of MT-1 and MT-2 were shown on the tail of one major peak, which migrated at about $3 \mathrm{~min}$. After $15 \mathrm{~s}$ incubation at $100{ }^{\circ} \mathrm{C}$, the baseline was found to be stable and many peaks disappeared. The peak heights of MT-1 and MT-2 decreased gradually by heat treatment. The peak migrated at $6.6 \mathrm{~min}$ decreased dramatically and the peak migrated behind MT-1 disappeared after $30 \mathrm{~s}$ incubation. The peak in front of MT2 disappeared after $60 \mathrm{~s}$ incubation. However, the ratio of peak heights of MT-1 and MT-2 did not change by heat treatment, especially until $30 \mathrm{~s}$ incubation (Tab. I).

\section{Discussion}

There are several methods for quantification of the MT content in tissues, such as Cd-hem, ELISA, and polarography $[1,3]$. Of these methods, Cd-hem method is a simple and useful for measurement of MT content in tissues without any instruments and chemicals, although atomic absorption spectrometer (AAS) is needed. Dieter et al. compared five methods, Cd-hem, Hg-saturation assay, radio immunoassay, thiolate group determination, and Sephadex G-75 gel chromatography with subsequent determination method by AAS observed that the recovery rate of MT contents measured by Cd-hem performed best [5]. The Cd-hem method assumes that MT is a heat stable protein [1,3,4]. MT is known to exist as four major isoforms and several sub-isoforms [1]. The isoforms MT-1 and MT-2 are generally

Table I. Effect of heat treatment on MT-1 and MT-2 isoforms in the cytosol fraction.

\begin{tabular}{|c|c|c|c|c|c|}
\hline \multirow{2}{*}{$\begin{array}{l}\text { Time for heat } \\
\text { treatment }\left(100{ }^{\circ} \mathrm{C}\right)\end{array}$} & \multicolumn{2}{|c|}{ Migration time (min) } & \multicolumn{2}{|c|}{ Peak intensity (abs) } & \multirow{2}{*}{$\begin{array}{c}\text { Intensity ratio } \\
\text { MT-2/MT-1 }\end{array}$} \\
\hline & MT-1 & MT-2 & $M T-1$ & MT-2 & \\
\hline None & 7.5 & 5.2 & 0.0075 & 0.0075 & 1.0 \\
\hline $15 \mathrm{sec}$ & 7.5 & 5.2 & 0.0071 & 0.0071 & 1.0 \\
\hline $30 \mathrm{sec}$ & 7.5 & 5.2 & 0.0066 & 0.0066 & 1.0 \\
\hline $1 \mathrm{~min}$ & 7.6 & 5.2 & 0.0053 & 0.0057 & 1.1 \\
\hline $2 \min$ & 7.7 & 5.2 & 0.0050 & 0.0054 & 1.1 \\
\hline $4 \mathrm{~min}$ & 7.6 & 5.2 & 0.0046 & 0.0050 & 1.1 \\
\hline
\end{tabular}


induced in the same organ by the same stimuli such as stress and heavy metals [3], whereas MT-3 and MT-4 are mainly loaded in the brain and stratified squamous epithelia $[7,8]$.

We previously separated the MT-1 and MT-2 isoforms in a cytosol fraction by CZE using a polyacrylamide-coated capillary at a neutral $\mathrm{pH}$. CZE is a powerful separation technique for the separation of the two isoforms of metallothionein MT-1 and MT-2 [10]. The standard and the real sample should contain the same metal in the perspective of the identification of the peaks in the electropherogram of the cytosol fraction on the basis of the migration time obtained for the standards MT-1 and MT-2. However, we used Cd, Zn-MT-1 as standard, $\mathrm{Cd}_{7}-\mathrm{MT}-1$ as purified isoforms and $\mathrm{Zn}_{7}$-MT as real samples. When these isoforms were identified by using anti-MT antibody on CZE analysis, the migration times of those samples coincided [11]. Therefore, in the present study, MT isoforms in the real samples were identified from those migration times corresponded with those of purified isoforms.

In the present study, the effect of heat treatment during a short period of time is a partial elimination of the majority of other proteins than MT isoforms, and the peaks of MT-1 and MT-2 in standard solution were stable in comparison with the isoforms in cytosol fraction. The reason why the peak heights of MT isoforms decreased after short-term incubation may be that unknown substances are migrated in the same positions of MT isoforms. However, the ratio of MT-1 and MT-2 in the cytosol fraction did not change by heat treatment, especially $30 \mathrm{sec}$ incubation. In addition, the substances migrated close by MT-1 and MT-2 disappeared after incubation for $60 \mathrm{~s}$.

In conclusion, majority of other proteins than MT isoforms can be deprived of the cytosol fraction by heat treatment during a short period of time, and the ratio of MT1 and MT-2 in the cytosol fraction on CZE analysis can be observed after the heat treatment within 1 min. However, it is not adequate for observation of properties of MT isoforms after heat treatment, as each peak of both MTs decreases by the treatment.

\section{References}

1. Sato, M.; Suzuki, K.T. Biomed. Res. Trace Elem. 1995, 6, 1328.

2. Moffatt, P.; Denizeau, F. Drug Metab. Rev. 1997, 29, 261-307.

3. Waalkes, M.P. In Endocrine Methods; Thomas, J.A., Ed. New York: Academic Press Inc. 1996; pp. 371-391.

4. Onosaka, S.; Tanaka, K.; Doi, M.; Okahara, K. Eisei Kagaku 1978, 24, 128-131.

5. Dieter, H.H.; Müller, L.; Abel, J.; Summer, K.-H. Toxicol. Appl. Pharmacol. 1986, 85, 380-388.

6. Kubo, K. Anal. Biochem. 1996, 241, 42-46.

7. Minami, T.; Yoshita, C.; Tanaka, M.; Kubo, K.; Okabe, N.; Okazaki, Y. Talanta 1998, 46, 347-354.

8. Aschner, M., Cherian, M.G., Klaassen, C.D., Palmiter, R.D., Erickson, J.C., Bush, A.I. Toxicol. Appl. Pharmacol. 1997, $142,229-242$.

9. Quaife, C.J., Findley, S.D., Erickson, J.C., Froelick, G.J., Kelly, E.J., Zambrowicz, B.P., Palmiter, R.D. Biochemistry 1994, 33, 7250-7259.

10. Minami, T.; Matsubara, H.; O-higashi, M.; Otaki, N.; Kimura, M.; Kubo, K.; Okabe, N.; Okazaki, Y. J. Chromatogr. B 1996, 685, 353-359.

11. Kubo, K., Sakita, Y., Okazaki, Y., Otaki, N., Kimura, M., Minami, T. J. Chromatogr. B 1999, 736, 185-190. 